\title{
The total deuterium abundance in the local Galactic disk: decisions and implications
}

\author{
Jeffrey L. Linsky ${ }^{1}$ \\ ${ }^{1}$ JILA, University of Colorado and NIST, \\ Campus Box 440, Boulder, CO 80309-0440, USA \\ email: jlinsky@jilau1.colorado.edu
}

\begin{abstract}
Analyses of FUSE spacecraft spectra have provided measurements of $\mathrm{D} / \mathrm{H}$ in the gas phase of the interstellar medium for many lines of sight extending to several kpc from the Sun. These measurements, together with the earlier Copernicus, HST, and IMAPS data, show a wide range of $\mathrm{D} / \mathrm{H}$ values that have challenged both observers and chemical evolution modellers. I believe that the best explanation for the diverse $\mathrm{D} / \mathrm{H}$ measurements is that deuterium can be sequestered on to carbonaceous grains and $\mathrm{PAH}$ molecules and thereby removed from the interstellar gas. Grain destruction can raise the gas phase $\mathrm{D} / \mathrm{H}$ value to approximately the total $\mathrm{D} / \mathrm{H}$ value. Supernovae and stellar winds, however, can decrease the total $\mathrm{D} / \mathrm{H}$ value along lines of sight on time scales less than mixing time scales. I will summarize the theoretical and observational arguments for this model and estimate the most likely range for the total $\mathrm{D} / \mathrm{H}$ in the local Galactic disk. This range in total $\mathrm{D} / \mathrm{H}$ presents a constraint on realistic Galactic chemical evolution models or the primordial value of $\mathrm{D} / \mathrm{H}$ or both.
\end{abstract}

Keywords. ISM: abundances; cosmology: cosmological parameters; ultraviolet: ISM

\section{Introduction}

It has long been recognized that accurate measurements of the primordial abundance of deuterium provides the best constraint on the properties of the early universe (1001000 seconds after the Big Bang) and, in particular, the ratio of baryons to photons $\left(\eta_{10}\right)$. Deuterium plays this critical role because it is an ideal relic; since its initial creation there has been no known process for creation of a significant amount of deuterium, but only destruction by nuclear reactions in stars. Analysis of quasar absorption lines (QAL) in seven lines of sight led Pettini et al. $(2008)$ to infer $(\mathrm{D} / \mathrm{H})_{\mathrm{QAL}}=28.2_{-1.8}^{+2.0}$ parts per million (ppm). Analysis of the cosmic microwave background signal leads to a measurement of $\eta_{10}$ and thus inference of the primordial $\mathrm{D} / \mathrm{H}$ ratio. In the most recent analysis of the 5 -year WMAP data set, Dunkley et al. (2009) obtain the parameter $100 \Omega_{b, 0}=2.273 \pm 0.062$, which corresponds to $\eta_{10}=6.225 \pm 0.0170$ and $(\mathrm{D} / \mathrm{H})_{\mathrm{WMAP}}=25.2 \pm 1.1$. While the two values for the primordial or near-primordial $\mathrm{D} / \mathrm{H}$ ratio, $(\mathrm{D} / \mathrm{H})_{\mathrm{QAL}}$ and $(\mathrm{D} / \mathrm{H})_{\mathrm{WMAP}}$, are consistent, they are sufficiently different and likely to be subject to different systematic errors that they should not be averaged to obtain a more "robust" value of $(D / H)_{\text {prim }}$.

Since the first measurements of the interstellar deuterium Lyman lines by the Copernicus satellite, there have been many attempts to measure accurate deuterium abundances in the interstellar medium of our Galaxy. These have included measurements of the deuterium and hydrogen Lyman- $\alpha$ line with IUE and HST, as well as measurements of the higher Lyman lines with the Interstellar Medium Absorption Profile Spectrograph (IMAPS). More recently, the Far Ultraviolet Spectrograph Explorer (FUSE) has provided many new deuterium measurements along lines of sight extending to nearly $3 \mathrm{pc}$. 
Until recently, the factor of four range in measured $\mathrm{D} / \mathrm{H}$ values for different lines of sight (Fig. 1) extending to stars beyond the Local Bubble posed a severe problem as no chemical evolution model of the Galaxy could explain this wide range. A solution to the problem proposed by Jura (1982) and developed by Draine (2003) and Draine (2006) is that deuterium is more tightly bound to carbonacious grains and PAH molecules than hydrogen because the $\mathrm{C}-\mathrm{D}$ bond is slightly larger than the $\mathrm{C}-\mathrm{H}$ bond. This is important because interstellar grains are cold $(T \sim 20 \mathrm{~K})$ and can thus sequester significant amounts of deuterium from the gas phase. Linsky et al. (2006) provided support for this explanation with three lines of evidence: (1) very high $\mathrm{D} / \mathrm{H}$ ratios measured in interstellar carbonaceous dust grains embedded in interplanetary dust particles (a proof of concept), (2) correlation of high $\mathrm{D} / \mathrm{H}$ values in interstellar gas with large depletions of refractory metals (Fig. 2), implying that deuterium and metals both deplete onto and evaporate from dust grains although the story is more complex, and (3) D/H abundances increase with interstellar gas temperature as inferred from excitation of $\mathrm{H}_{2}$. Thus the total $\mathrm{D} / \mathrm{H}$ ratio in the interstellar medium $(\mathrm{D} / \mathrm{H})_{\text {total }}$ can be significantly larger than the gas phase measurements $(\mathrm{D} / \mathrm{H})_{\text {gas }}$. Since even in recently shocked gas, some of the grains containing deuterium may still be present, one must conclude that $(\mathrm{D} / \mathrm{H})_{\text {total }} \geqslant(\mathrm{D} / \mathrm{H})_{\text {gas }}$. This statement is strengthened by recognizing that lines of sight likely include both recently shocked and unshocked gas containing grains with deuterium.

Many authors have now accepted the deuterium-depleted dust hypothesis as the explanation for the measured low values of $(\mathrm{D} / \mathrm{H})_{\text {gas }}$. In this talk I would like to address the high values of $(\mathrm{D} / \mathrm{H})_{\text {gas }}$ including recent measurements, infer a sensible value for

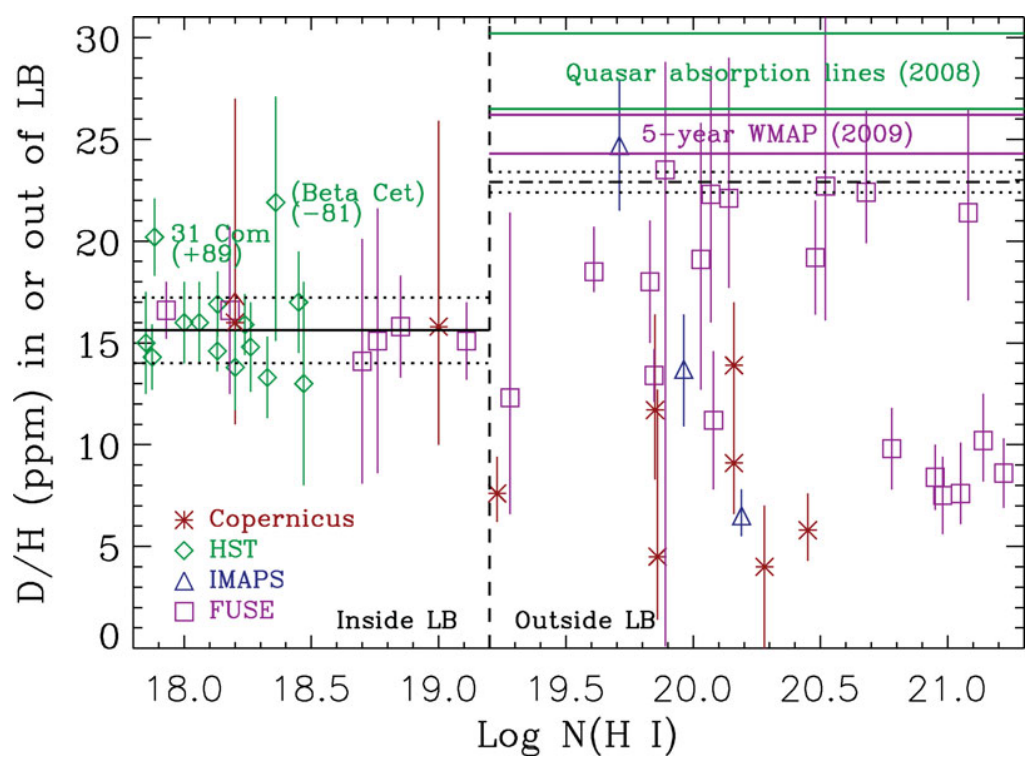

Figure 1. Measurements of the $(\mathrm{D} / \mathrm{H})_{\text {gas }}$ for the 24 lines of sight located entirely inside of the Local Bubble (to the left of the vertical dashed line) and the gas located outside of the Local Bubble, $(\mathrm{D} / \mathrm{H})_{\text {gas }-\mathrm{LB}}$, (to the right of the vertical dashed line). The error bars are $1 \sigma$, and the horizontal axis is the hydrogen column density to the star at the end of the line of sight. The symbols are coded by the spacecraft that obtained the Lyman line spectra. The range of $(\mathrm{D} / \mathrm{H})_{\text {prim }}$ values obtained from quasar absorption lines (Pettini et al. 2008) and from WMAP (Dunkley et al. 2009) are shown in the upper right. Just below is the range of the total deuterium abundance in the local Galactic disk, $(\mathrm{D} / \mathrm{H})_{\mathrm{high}}=22.9 \pm 0.5 \mathrm{ppm}$. 
$(\mathrm{D} / \mathrm{H})_{\text {total }}$ in the Galactic disk, and relate this high value of $(\mathrm{D} / \mathrm{H})_{\text {total }}$ to the measurements of primordial $\mathrm{D} / \mathrm{H}$.

\section{Analysis of the high values of $(\mathrm{D} / \mathrm{H})_{\text {gas }}$}

Linsky et al. (2006) assembled all of the then-available published values of $(\mathrm{D} / \mathrm{H})_{\mathrm{gas}}$ obtained with Copernicus, IUE, HST, IMAPS, and FUSE for lines of sight toward 47 stars. Since that paper was published, $(\mathrm{D} / \mathrm{H})_{\text {gas }}$ measurements for five additional lines of sight observed by FUSE have became available (Table1) and are included in the following analysis. I consider only the direct measurements of $\mathrm{N}(\mathrm{HI})$ and $\mathrm{N}(\mathrm{DI})$ for which the hydrogen and deuterium components of the molecules $\mathrm{H}_{2}$ and $\mathrm{HD}$ are added when measured. There are alternative methods for evaluating $\mathrm{D} / \mathrm{H}$, in particular from the product $(\mathrm{D} / \mathrm{O}) \mathrm{x}(\mathrm{O} / \mathrm{H})$. As described by Hébrard (2010), this method minimizes errors in $\mathrm{N}(\mathrm{HI})$ due to the high opacity of the Lyman lines. However, this method introduces additional uncertainties associated with measurements of the oxygen lines, including depletion of oxygen on interstellar dust grains.

Linsky et al. (2006) separated the $(\mathrm{D} / \mathrm{H})_{\text {gas }}$ measurements into two groups depending upon whether the target star is located inside of or beyond the Local Bubble. The Local Bubble has been described as a superbubble of $10^{6} \mathrm{~K}$ gas produced by recent supernovae in the Scorpio-Centaurus Association of young stars, although Welsh \& Shelton (2009) argue that most of the volume of the gas is highly ionized but far cooler than $10^{6} \mathrm{~K}$. The $\mathrm{N}(\mathrm{HI})$ and $\mathrm{N}(\mathrm{DI})$ measurements toward stars inside of the Local Bubble refer to warm gas (4,000-10,000 K) located in clouds near the Sun (Redfield \& Linsky 2008). The Local Bubble is mostly surrounded by cold gas detected in NaI beginning at about $\log \mathrm{N}(\mathrm{HI})=19.2$, but there is no cold gas near the Galactic poles (Fig. 3) allowing the Local Bubble gas to interact with the halo. Most lines of sight to the 24 stars located inside of the Local Bubble show $(\mathrm{D} / \mathrm{H})_{\text {gas }}$ ratios consistent with the mean value of

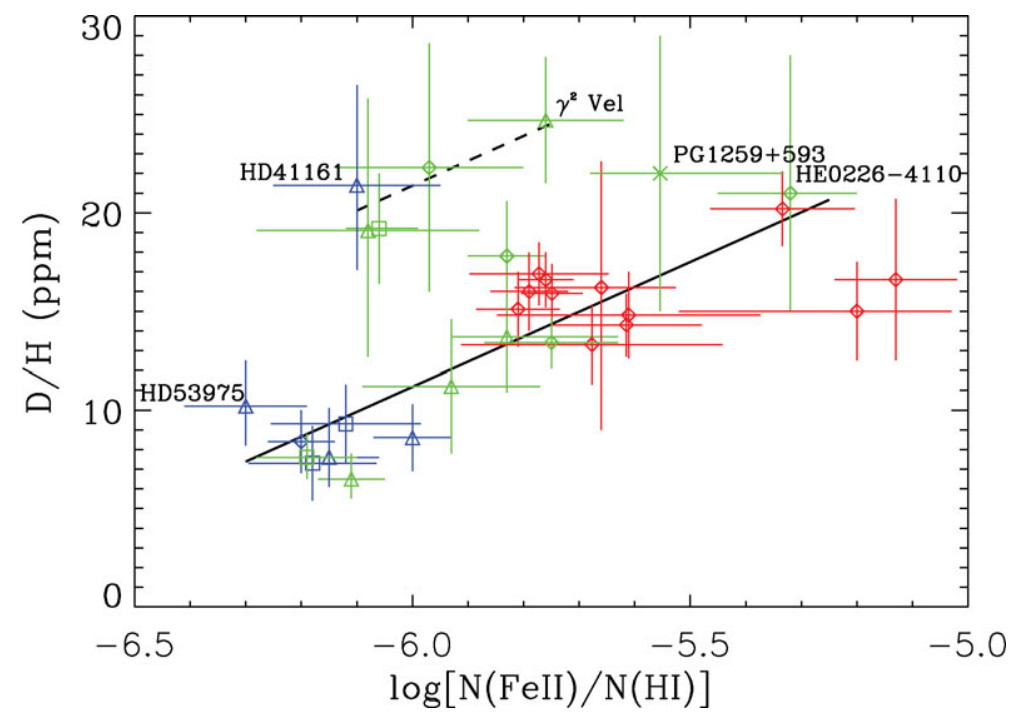

Figure 2. Plot of $(\mathrm{D} / \mathrm{H})_{\text {gas }}$ vs. depletion of iron for the lines of sight observed by HST, IMAPS, and FUSE. The coding symbols are the same as in Fig. 1. The solid line is the best fit to all of the data, and the dashed line is a displacement of this line upward by $8.5 \mathrm{ppm}$ to be consistent with the five data points that lie well above the mean fit line. The five high points may be lines of sight where weak shocks have removed deuterium, but not iron, from the grains. 
Table 1. Recent measurements of $(\mathrm{D} / \mathrm{H})_{\text {gas }}^{1}$.

\begin{tabular}{lccccccc}
\hline Target & $l$ & $b$ & $d(p c)$ & $\log \mathrm{N}(\mathrm{HI})^{2}$ & $\log \mathrm{N}(\mathrm{DI})^{3}$ & $(\mathrm{D} / \mathrm{H})_{\mathrm{gas}}$ & $(\mathrm{D} / \mathrm{H})_{\mathrm{gas}-\mathrm{LB}}$ \\
\hline REJ 1738+665 & 97 & +32 & 243 & $19.83 \pm 0.05$ & $15.08 \pm 0.04$ & $17.8_{-2.5}^{+2.8}$ & $18 \pm 3$ \\
HD 41161 & 165 & +13 & 1253 & $21.08 \pm 0.08$ & $16.41 \pm 0.05$ & $21.4_{-4.3}^{+5.1}$ & $21.4_{-4.3}^{+5.1}$ \\
HD 53975 & 226 & -02 & 1318 & $21.14 \pm 0.06$ & $16.15 \pm 0.07$ & $10.2_{-2.0}^{+2.3}$ & $10.2_{-2.0}^{+2.3}$ \\
HD 93521 & 183 & +62 & & $19.61 \pm 0.055$ & $14.85_{-0.02}^{+0.05}$ & $17.4_{-0.8}^{+2.0}$ & $18.5_{-1.0}^{+2.2}$ \\
LSE 234 & 329 & -21 & $460 \pm 120$ & $20.68_{-0.05}^{+0.025}$ & $16.02 \pm 0.045$ & $22.0_{-2.5}^{+4}$ & $22.4_{-2.5}^{+4}$ \\
\hline
\end{tabular}

Notes:

${ }^{1}$ All errors are $\pm 1 \sigma$. References: Dupuis et al. (2009) for REJ 1738+665, Oliveira \& Hébrard (2006) for HD 41161 and HD 53975, Kruk et al. (2006) for HD 93521, and Lecavelier des Etangs et al. (2006) for LSE 234. ${ }^{2} \mathrm{~N}(\mathrm{HI})$ includes $2 \mathrm{~N}\left(\mathrm{H}_{2}\right)$ and $\mathrm{N}(\mathrm{HD})$.

${ }^{3} \mathrm{~N}(\mathrm{DI})$ includes $\mathrm{N}(\mathrm{HD})$.

${ }^{4} \mathrm{HD} 93521$ is located in the Galactic halo at $\mathrm{z}=1.5 \mathrm{kpc}$.

$15.6 \pm 0.4 \mathrm{ppm}$, but the two highest points toward $31 \mathrm{Com}$ and $\beta$ Cet are for stars located at high Galactic latitudes $\left(+89^{\circ}\right.$ and $\left.-81^{\circ}\right)$. I include the line of sight to 31 Com in the Local Bubble measurements as the $\mathrm{N}(\mathrm{HI})$ and $\mathrm{N}(\mathrm{DI})$ gas in this line of sight has a velocity consistent with the North Galactic Pole cloud of warm gas located within $8.5 \mathrm{pc}$ of the Sun (Redfield \& Linsky 2008). I also include the line of sight to $\beta$ Cet in the Local Bubble measurements as the velocities of the $\mathrm{H}(\mathrm{I})$ and $\mathrm{D}(\mathrm{I})$ gas are consistent with the Microscopium and Ceti clouds located within 5.1 and $15.5 \mathrm{pc}$ of the Sun.

Since the inner boundary of the Local Bubble lies at $\log \mathrm{N}(\mathrm{HI}) \approx 19.2$ (Sfeir et al. 1999, Lallement et al. 2003) in nearly all directions, it is sensible to subtract the Local Bubble foreground from the column densities toward stars that lie beyond the Local Bubble to infer $(\mathrm{D} / \mathrm{H})_{\text {gas }}$ ratios for the ISM not modified by deuterium-depleted gas from the Local Bubble supernovae. Assuming $(\mathrm{D} / \mathrm{H})_{\text {gas }}=15.6 \pm 0.4 \mathrm{ppm}$, the Local Bubble foreground is $\log \mathrm{N}(\mathrm{HI})_{\mathrm{LB}}=19.20$ and $\log \mathrm{N}(\mathrm{DI})_{\mathrm{LB}}=14.39$. The ratios $(\mathrm{D} / \mathrm{H})_{\text {gas }-\mathrm{LB}}$ in Table 1 and in Table 3 of Linsky et al. (2006) include this correction. The effect of subtracting the Local Bubble foreground is to produce somewhat higher or lower values of $(\mathrm{D} / \mathrm{H})_{\mathrm{gas}-\mathrm{LB}}$ for lines of sight toward stars just beyond the Local Bubble when $(\mathrm{D} / \mathrm{H})_{\text {gas }}$ is greater or less than $15.6 \mathrm{ppm}$. The correction is small for stars with $\log \mathrm{N}(\mathrm{HI})>20.5$.

In Fig. 1, ten lines of sight have $(\mathrm{D} / \mathrm{H})_{\text {gas }-\mathrm{LB}}>15.6$, and six lie above 20.0. If the deuterium-depletion hypothesis is the major cause of low values of $(\mathrm{D} / \mathrm{H})_{\mathrm{gas}-\mathrm{LB}}$, then
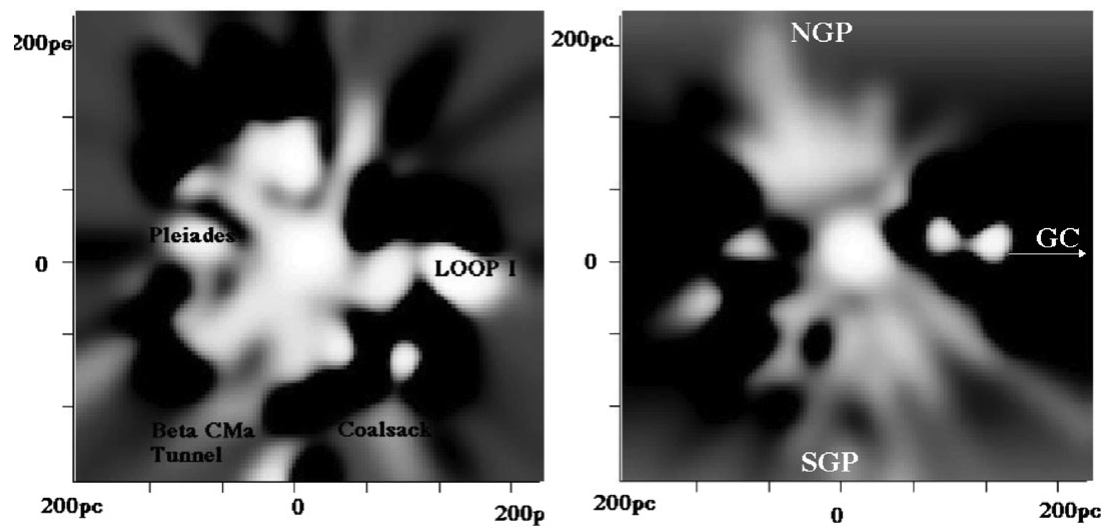

Figure 3. Two views of the Local Bubble from Welsh (2009) and Lallement et al. (2003). Dark indicates cold gas seen in the NaI, and white indicates no cold gas. Left: view of the Galactic plane from the North Galactic pole. Right: view from Galactic longitude $270^{\circ}$ and latitude $0^{\circ}$. 
the highest credible value or values of $(\mathrm{D} / \mathrm{H})_{\text {gas }-\mathrm{LB}}$ should be the best approximation to a lower limit to $(\mathrm{D} / \mathrm{H})_{\text {total }}$. Given that the data have errors, one can only estimate the highest credible value of $(\mathrm{D} / \mathrm{H})_{\text {gas }-\mathrm{LB}}$ statistically. At this symposium, Steigman (2010), has proposed another statistical method for estimating $(\mathrm{D} / \mathrm{H})_{\text {total }}$. In Fig. 4, I plot the value of $(\mathrm{D} / \mathrm{H})_{\text {high }}$ estimated as the mean of the $(\mathrm{D} / \mathrm{H})_{\text {gas }-\mathrm{LB}}$ values starting with the highest value and sequentially including lower values and weighting each data point by the inverse errors. Also plotted is $\sigma$, the error of the mean for each number of lines of sight included. The value of $\sigma$ goes through a minimum at seven lines of sight and increases thereafter. For these seven lines of sight, $(\mathrm{D} / \mathrm{H})_{\mathrm{high}}=22.9 \pm 0.5 \mathrm{ppm}$. I therefore propose that the total $\mathrm{D} / \mathrm{H}$ ratio (gas plus dust) in the Galactic disk near the Sun is $(\mathrm{D} / \mathrm{H})_{\text {total }} \geqslant 22.9 \pm 0.5 \mathrm{ppm}$.

\section{Decisions and implications}

Comparison of our estimate of the total $\mathrm{D} / \mathrm{H}$ ratio in the nearby Galactic disk outside of the Local Bubble with estimates of the primordial $\mathrm{D} / \mathrm{H}$ ratio places constraints on the evolution of deuterium over the lifetime of the Galaxy. Models of the chemical evolution of the Galaxy (e.g., Romano et al. 2006) include both the destruction of deuterium by nuclear reactions in stars and estimates of the infall of gas from captured galaxies and the intergalactic medium containing near primordial D/H. For different assumptions, these models compute the deuterium astration factor, $f_{\mathrm{D}}=(\mathrm{D} / \mathrm{H})_{\operatorname{prim}} /(\mathrm{D} / \mathrm{H})_{\mathrm{high}}$. In Table 2 , I list $f_{\mathrm{D}}$ computed from the QAL and WMAP values for $(\mathrm{D} / \mathrm{H})_{\text {prim }}$. Also, given are the corresponding fractions of deuterium atoms today in the nearby Galactic disk that have not been processed through stars.

The values of $f_{\mathrm{D}}$ listed in the table computed from the QAL value of $(\mathrm{D} / \mathrm{H})_{\text {prim }}$ and especially the WMAP value challenge recent Galactic chemical evolution models for the galactocentric distance of the Sun. The models of Chiappini et al. (2002), for example, predict that $f_{\mathrm{D}} \approx 1.5$ for a wide range of assumptions. The more recent models of Romano et al. (2006) can accommodate values of $f_{\mathrm{D}}$ as low as $\sim 1$. While there may

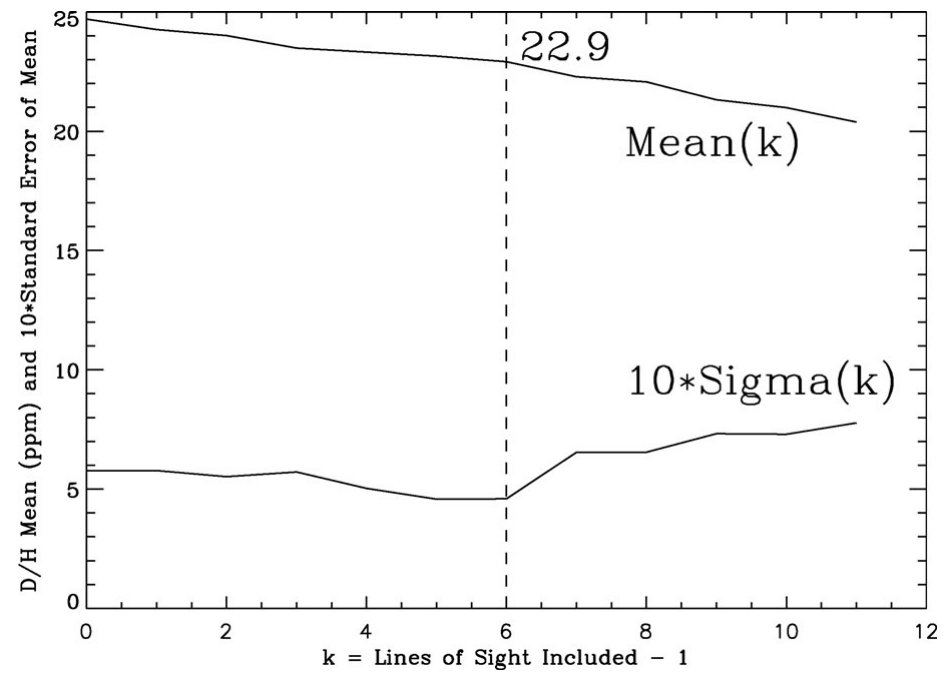

Figure 4. Mean values of $(\mathrm{D} / \mathrm{H})_{\text {high }}$ obtained as a weighted average of $(\mathrm{D} / \mathrm{H})_{\mathrm{gas}-\mathrm{LB}}$ values starting with the highest and including next lower values in sequence. The error in the mean, $\sigma$, increases above seven lines of sight, implying that the best value of the total $\mathrm{D} / \mathrm{H}$ ratio in the local Galactic disk is $(\mathrm{D} / \mathrm{H})_{\mathrm{high}} \geqslant 22.9 \pm 0.5 \mathrm{ppm}$. 
Table 2. Is Concordance Possible?

\begin{tabular}{lccccc}
\hline Method & D / H $(\mathbf{p p m})$ & $100 \Omega_{b, 0} h^{2}$ & $\eta_{10}$ & $f_{\mathrm{D}}$ & Fraction \\
\hline ISM (high 7) & $\geqslant 22.9 \pm 0.5$ & $\leqslant 2.411 \pm 0.33$ & $\leqslant 6.604 \pm 0.090$ & & \\
QAL (7 LOS) & $28.2_{-1.8}^{+2.0}$ & $2.13 \pm 0.09$ & $5.84 \pm 0.27$ & $\leqslant 1.23 \pm 0.09$ & $\geqslant 81 \pm 7 \%$ \\
WMAP (5 years) & $25.2 \pm 1.1$ & $2.273 \pm 0.062$ & $6.225 \pm 0.170$ & $\leqslant 1.10 \pm 0.07$ & $\geqslant 91 \pm 6 \%$ \\
\hline
\end{tabular}

not be an inconsistency with the QAL data, the WMAP value for $f_{\mathrm{D}}$ severely challenges even the Romano et al. (2006) models. At this point, it is not clear to me which are the major causes for the inconsistency, but important unknowns are the accretion rate of near primordial gas and the time scale for mixing of this gas with the ISM. Prodanovic $\&$ Fields (2008) argue that an accretion rate of $\sim 1 M_{\odot} \mathrm{yr}^{-1}$ could explain the high percentage of unprocessed deuterium. This topic is becoming increasingly interesting.

\section{References}

Chiappini, C., Renda, A., \& Matteucci, F. 2002, A\& $A$, 395, 789

Draine, B. T. 2003, ARAA, 41, 241

Draine, B. T. 2006, in A. McWilliam \& M. Rauch (eds.), Origin and Evolution of the Elements, (Cambridge Univ. Press), p. 317

Dunkley, J. et al. 2009, ApJS, 180, 306

Dupuis, J., Oliveira, C. M., Hébrard, G. H., Moos, H. W., \& Sonnentrucker, P. 2009, ApJ, 690, 1045

Hébrard, G. 2010, in C. Charbonnel, M. Tosi, F. Primas, \& C. Chiappini (eds), Light Elements in the Universe (Cambridge Univ. Press), this volume

Jura, M. 1982, in Y. Kondo, J. M. Meade, \& R. D. Chapman (eds), Advances in UV Astronomy: 4 Years of IUE Research, (NASA CP 2238), p. 54

Kruk, J. W., Oliveira, C., Sembach, K. R., \& Savage, B. D. 2006, in G. Sonneborn, H. W. Moos, \& B.-G. Andersson (eds), Astrophysics in the Far Ultraviolet, Five Years of Discovery with FUSE, ASP-CS, 348 , p. 85

Lallement, R., Welsh, B., Vergeley, J. L., Crifo, F., \& Sfeir, D. 2003, A\&AA, 411, 447

Lecavelier des Etangs, A., Hébrard, G., \& Williger, G. M. in G. Sonneborn, H. W. Moos, \& B.-G. Andersson (eds), Astrophysics in the Far Ultraviolet, Five Years of Discovery with FUSE, ASP-CS, 348, p. 88

Linsky, J. L. et al. 2006, ApJ, 647, 1106

Oliveira, C. M. \& Hébrard, G. 2006, ApJ, 653, 345

Pettini, M., Zych, B. J., Murphy, M. T., Lewis, A., \& Steidel, C. C. 2008, MNRAS, 391, 1499

Redfield, S. \& Linsky, J. L. 2008, ApJ, 673, 283

Prodanović, T. \& Fields, B. D. 2008, J. Cosmology \& Astroparticle Physics, 09, 003

Romano, D., Tosi, M., Chiappini, C., \& Matteucci, F. 2006, MNRAS, 369, 295

Sfeir, D. M., Lallement, R., Crifo, F., \& Welsh, B. Y. 1999, A\&A, 346, 785

Steigman, G. 2010, in C. Charbonnel, M. Tosi, F. Primas, \& C. Chiappini (eds), Light Elements in the Universe (Cambridge Univ. Press), this volume

Welsh, B. Y. \& Shelton, R. L. 2009, APESS , 323, 1 